\title{
Intra- and inter-group coordination patterns reveal collective behaviors of football players near the scoring zone
}

\author{
Ricardo Duarte $^{\mathrm{a}, *}$, Duarte Araújo ${ }^{\mathrm{a}}$, Luís Freire ${ }^{\mathrm{a}}$, Hugo Folgado ${ }^{\mathrm{b}}$, \\ Orlando Fernandes ${ }^{b}$, Keith Davids ${ }^{c}$
}

a Faculty of Human Kinetics, Technical University of Lisbon, Portugal

${ }^{\mathrm{b}}$ School of Science and Technology, University of Évora, Portugal

'School of Human Movement Studies, Queensland University of Technology, Australia

\section{A R T I C L E I N F O}

\section{Article history:}

Available online 17 April 2012

\section{PsycINFO classification:}

3020

3720

Keywords:

Group performance

Geometrical center

Surface area

Coordination tendencies

Association Football

\begin{abstract}
A B S T R A C T
This study examined emergent coordination processes in collective patterns of behavior in 3 vs 3 sub-phases of the team sport of association football near the scoring zone. We identified coordination tendencies for the centroid (i.e., team center) and surface area (i.e., occupied space) of each sub-group of performers $(n=20$ plays). We also compared these kinematic variables at three key moments of play using mixed-model ANOVAs. The centroids demonstrated a strong symmetric relation that described the coordinated attacking/defending actions of performers in this sub-phase of play. Conversely, analysis of the surface area of each team did not reveal a clear coordination pattern between subgroups. But the difference in the occupied area between the attacking and defending sub-groups significantly increased over time. Findings emphasized that major changes in sub-group behaviors occurred just before an assisted pass was made (i.e., leading to a loss of stability in the 3 vs 3 sub-phases).
\end{abstract}

(C) 2012 Elsevier B.V. All rights reserved.

\section{Introduction}

The study of collective system behaviors in nature has contributed to understanding of how large populations of organisms interact and adapt their actions to achieve common goals (e.g., Deneubourg \& Goss, 1989). For example, initial studies of biological systems have revealed spontaneous

\footnotetext{
* Corresponding author. Address: Faculty of Human Kinetics, Technical University of Lisbon, Estrada da Costa, 1495-688 Cruz Quebrada, Portugal. Tel.: +351 214149166.

E-mail address: rduarte@fmh.utl.pt (R. Duarte).
} 
(i.e., non-externally controlled), emergent collective behaviors in schools of fish (Partridge, 1982; Partridge \& Pitcher, 1979), swarms of honeybees (Visscher \& Camazine, 1999) and ant colonies (Mallon, Pratt, \& Franks, 2001; Pratt, Mallon, Sumpter, \& Franks, 2002). Similar lawful processes have been revealed in studies of human social systems such as waves produced by crowds at large sporting events, pedestrian escape panic (Farkas \& Vicsek, 2005) or traffic flows (Yuan, Wang, Xu, \& Li, 2005). In line with these findings, previous research has suggested that sports teams can also be regarded as complex, open systems (Davids, Araújo, \& Shuttleworth, 2005; Gréhaigne, Bouthier, \& David, 1997; McGarry, 2005), constituted of many degrees of freedom that result from the variety of possible interactions among system components (e.g., the attacker and defender interactions in a team game) (Davids, Button, \& Bennett, 2008; McGarry, Anderson, Wallace, Hughes, \& Franks, 2002). In these systems, collective behaviors emerge from the patterns of interpersonal coordination between agents (Bar-Yam, 2004; Passos et al., 2009), as a result of exchanges in energy, matter and information between them (e.g., team players) and their environment (e.g., the performance surroundings that constrain the behaviors of the performers) (Beek, Peper, \& Stegeman, 1995; Kugler, Kelso, \& Turvey, 1980).

Previous research on movement coordination has tended to emphasize the analysis of bivariate signals at the within-individual level (e.g., the movement oscillations of two index fingers, Kelso, 1984). In this regard, relative phase was the most prevalent coordinative variable used to assess coordination (for an application to interpersonal coordination in racket sports see Palut \& Zanone, 2005). However, the measurement of coordination processes in systems composed of three or more individuals (multivariate data) implies the need for a different strategy to capture and synthesize the state of a complex system.

From a coordination dynamics perspective, Schöllhorn (2003) has proposed some group-motion (kinematic) variables for analyzing team sports, such as the surface area occupied by players, the geometrical shape and geometrical center/or centroid of particular sub-groups of teams. This type of compound physical variables may be useful to synthesize and capture a system's low-dimensional dynamics, including social emergent collective behaviors. At this time, few studies have investigated coordination processes when more than two athletes interact over time during team sport performance. Lames, Erdmann, and Walter (2010) calculated the centroid and the ranges (in depth and width dimensions) of sports teams to study the spatiotemporal interactions of individuals during competitive performance. They observed a stable synchronization between performers in opposing teams with few and small perturbations throughout the game. They concluded that the tight spatiotemporal coupling observed between opposing performers evidenced the dependency and mutuality between the two teams studied, and that further work was needed to identify key events that disrupted stability of the coordination between teams. Frencken and colleagues (Frencken \& Lemmink, 2008; Frencken, Lemmink, Delleman, \& Visscher, 2011) analyzed centroid and surface area measures to capture the collective behaviors of teams in 4 vs 4 small-sided football games. They confirmed that measurement of team centroids accurately captured the synchronized tendencies between opposing teams. These investigators reported that the variable occupied surface area did not seem to adequately describe the interaction between opposing teams during competition. However, in some performance contexts there may be some intra-team coordination trends for surface area in these sub-group relations over time. In other words, observed variations in surface area may express intra-team coordination processes as a consequence of cooperative goal-directed behaviors (e.g., a number of teammates coordinating together to create a goal-scoring opportunity). A deeper understanding about the potential utility of these group-motion variables to establish the properties of social complex systems, such as sports teams, is needed. This work might contribute to the later identification of coordination variables in team sports and also in a wide range of other social collectives (Beek, Verschoor, \& Kelso, 1997).

Apart from these few studies of intra- and inter-team coordination processes in team sports, notational analysis research has revealed that $75-80 \%$ of shots at goal in elite international football competitions emerge from short passing sequences (Hughes \& Franks, 2005). These passing sequences typically involve a reduced number of players acting in localized sub-units trying to break the stability of interactions with the opposing team. Together, these data emphasize the need to investigate the collective behaviors of particular sub-groups of players involved in the creation/prevention of goalscoring opportunities in the team sport of association football. However, an apparent lack of data in performance contexts other than elite competitions exists. Therefore, the purpose of this paper was to investigate how collective behaviors emerge in 3 vs 3 sub-phases of intermediate-level youth 
football near the scoring zone. To achieve this purpose we identified coordination tendencies for the centroid and surface area of each team. Then, we compared these group-motion variables in three key moments of play, to understand their temporal evolution and clarify the intra- and inter-group coordination tendencies developed by the two sub-groups of performers.

\section{Methods}

\subsection{Participants}

Fourteen, male football players (age: $M=11.8, S D=0.4$ yrs; training experience: $M=3.6, S D=1.1 \mathrm{yrs}$ ) participated in the study. These participants were recruited from the same U13 team, which in accordance with Association Football regulations usually played competitively in a 7-a-side game format. Participants were selected due their intermediate level of skill, to avoid too experienced and idiosyncratic footballers, or inexperienced individuals who could not execute actions skilfully. All players and their parents were informed about the procedures and voluntarily agreed to participate in the study.

\subsection{Experimental task}

The experimental task was designed to be representative of a sub-phase of play involving creation of scoring opportunities near the scoring zone, typical of a 7-a-side competitive match for this age group. In this performance context, many shooting opportunities are created by disrupting the stability of the relative positioning between attacking and defending players in a 3 vs 3 sub-phase of performance near a scoring zone (McAvoy, 1998). Therefore, the experimental task consisted of a 3 vs 3 game, where in order to shoot at goal, the attacking performers needed to make a penetrating pass into the defensive space behind the defending players (see Fig. 1). The central space in the field of play was $20 \times 20 \mathrm{~m}$, and both defensive spaces were $14.5 \mathrm{~m}$ to simulate the goalkeeper's area. The defensive line was used to simulate the task constraints of the 7-a-side off-side rule for this age level. The twelve outfield players were divided into four separate teams. Four games of 5 min each were performed by the participants, with 3 min of passive recovery between games (Rampinini et al., 2007).

\subsection{Procedures}

Participants' movement displacement trajectories were captured by a statical digital video camera. For detailed analysis, we randomly selected 20 plays: (i) that ended in shots at goal, (ii) in which the

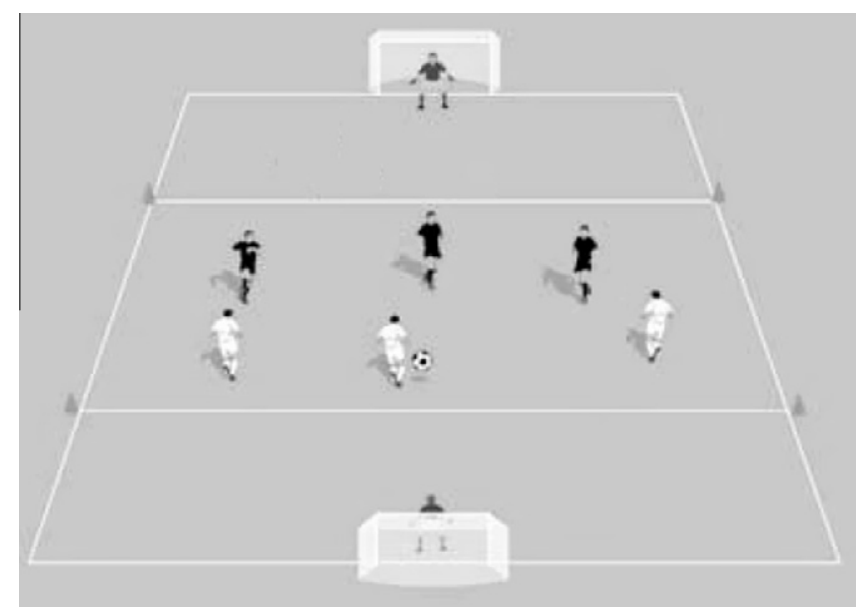

Fig. 1. Experimental task schematic representation. 
ball did not displace in an aerial trajectory, and (iii), which did not involve changes in ball possession between teams. These passages of performance represent short sequences in which the attacking team rapidly got into the scoring area after regaining ball possession. Video recordings were transferred and divided into 20 .avi files, corresponding to the selected plays. The beginning of the plays corresponded to the first touch of the attacking player who regained ball possession. The end of the play corresponded to the moment where the player who entered the scoring area touched the ball. For image treatment and to extract positional data from participants' movement displacement trajectories, we used a dedicated software package, TACTO 8.0, with accuracy levels reported as superior to 95\% (Fernandes, Folgado, Duarte, \& Malta, 2010). Camera calibration was made by comparing virtual (pixels units) and real measures (metric units) of seven control points. Next, the $x$ and $y$ virtual coordinates of the players were extracted with a data sampling rate of $25 \mathrm{~Hz}$. To transform the virtual into real coordinates we used the bi-dimensional Direct Linear Transformation method (2D-DLT) (Abdel-Aziz \& Karara, 1971). Detailed information about these time-motion analysis procedures is presented in the Appendix. The $x$ and $y$ coordinates were subsequently filtered with a Butterworth low pass filter $(6 \mathrm{~Hz})$ (Winter, 2005). To ensure appropriate quality control of measurements, the digitizing researcher undertook seven days of a digitization training program, consisting of digitizing two random plays per day (i.e., six outfield players involved in each play). On the seventh day, the researcher digitized the same play twice, interspersed by a break of five hours. In order to assess the intra-digitizer reliability we used the 'variation accounted for' measure (VAF) (Moorhouse \& Granata, 2007). Results showed high levels of reliability in calculating the displacements of the six digitized players in the $x$ - and $y$ component of motion (VAF always $>99.98 \%$ ) (for further details about these time-motion analysis procedures see Duarte et al., 2010). Subsequently, the centroid and surface areas of both teams in each play were computed using MATLAB software R2008a (The MathWorks, Inc., Natick, MA). The centroid of each team was calculated as the mean position of the three players over time in the $x$ - and $y$-component of motion. Next, we determined the smallest distance of the centroid to the defensive line using $x$-component motion values. The surface area of each team was calculated as the area of a triangle with the following formula for Cartesian coordinates:

$$
\operatorname{Area}(\mathrm{A}, \mathrm{B}, \mathrm{C})=\operatorname{abs}((x \mathrm{~B} * y \mathrm{~A}-x \mathrm{~A} * y \mathrm{~B})+(x \mathrm{C} * y \mathrm{~B}-x \mathrm{~B} * y \mathrm{C})+(x \mathrm{~A} * y \mathrm{C}-x \mathrm{C} * y \mathrm{~A})) / 2
$$

\subsection{Data analysis}

To analyze the spatiotemporal interactions between participants in attacking and defending teams, we used a running correlation technique (Araújo, Davids, \& Hristovski, 2006; Corbetta \& Thelen, 1996; Meador, Ray, Echauz, Loring, \& Vachtsevanos, 2002) that was applied along the entire data time-series recorded for each kinematic variable (i.e., the centroids and surface areas). We used a 0.4-s sliding window (i.e., a 10 data-point window) that was shifted frame by frame (i.e., every $0.04 \mathrm{~s}$ ). At every shift of the window, a correlation value was calculated, which resulted in a continuous correlation function that independently described the coordination of both teams for the centroid and surface area measurements over time (for a detailed description of this technique see Meador et al. (2002)). With running correlations we were able to identify three types of coordination tendencies (Corbetta \& Thelen, 1996): (i) symmetric patterns when the kinematic variables were predominantly correlated around high positive values; (ii) anti-symmetric patterns when kinematic variables displayed prevalent correlation frequencies around low negative values; and (iii) no correlated patterns when the values did not show a clear tendency for high or low associations.

In order to compare mean values of centroid and surface area of both sub-groups of performers (between-teams factor) at key moments of plays (within-teams factor), we used mixed-model ANOVA. Based on expert coaching knowledge (McAvoy, 1998) we identified three key moments in all the plays: (i) first touch of ball control by the player making the final pass in the move, (ii) last touch in the assisted pass made by the same player, and (iii) time of ball crossing the defensive line. Tukey's HSD test was used to discriminate mean differences in multiple comparisons. Data analyses were conducted using SPSS 17.0 (SPSS, Inc., Chicago, IL). Alpha levels were maintained at $p<.05$ for all statistical procedures. 


\section{Results}

The average duration of plays was $4.7 \pm 2.0 \mathrm{~s}$, with a mean of $2.9 \pm 1.8$ touches per player and $3.4 \pm 2.0$ passes per play. Exemplar data from these sequences of play were selected in order to illustrate the relation between the three key moments identified and the evolution over time of the groupmotion variables (i.e., centroid and surface area). Thereafter, the correlation landscapes of all trials show how the centroid and surface areas of both teams evolved and interacted over time. Finally, we provide additional results from mixed-model ANOVAs that compared the kinematic variables within- and between-teams during the plays.

\subsection{Coordination tendencies in 3 vs 3 sub-phases}

The upper panel of Fig. 2 describes the distance of the centroid of each team to the defensive line in a random selected play, as the game evolved in the playing field over time. The time of the three key events has been highlighted. A uniform decrease in the distance of the centroid to the defensive line was observed as a function of time. The bottom left panel shows the very stable corresponding correlation function that indicated a predominance of a symmetric pattern of coordination between the two sub-groups of performers. This observation signifies that both sub-groups moved forward and backward in a highly synchronized spatiotemporal manner. The bottom right panel displays the frequency histogram that strengthens the predominance of high correlation values during this play.

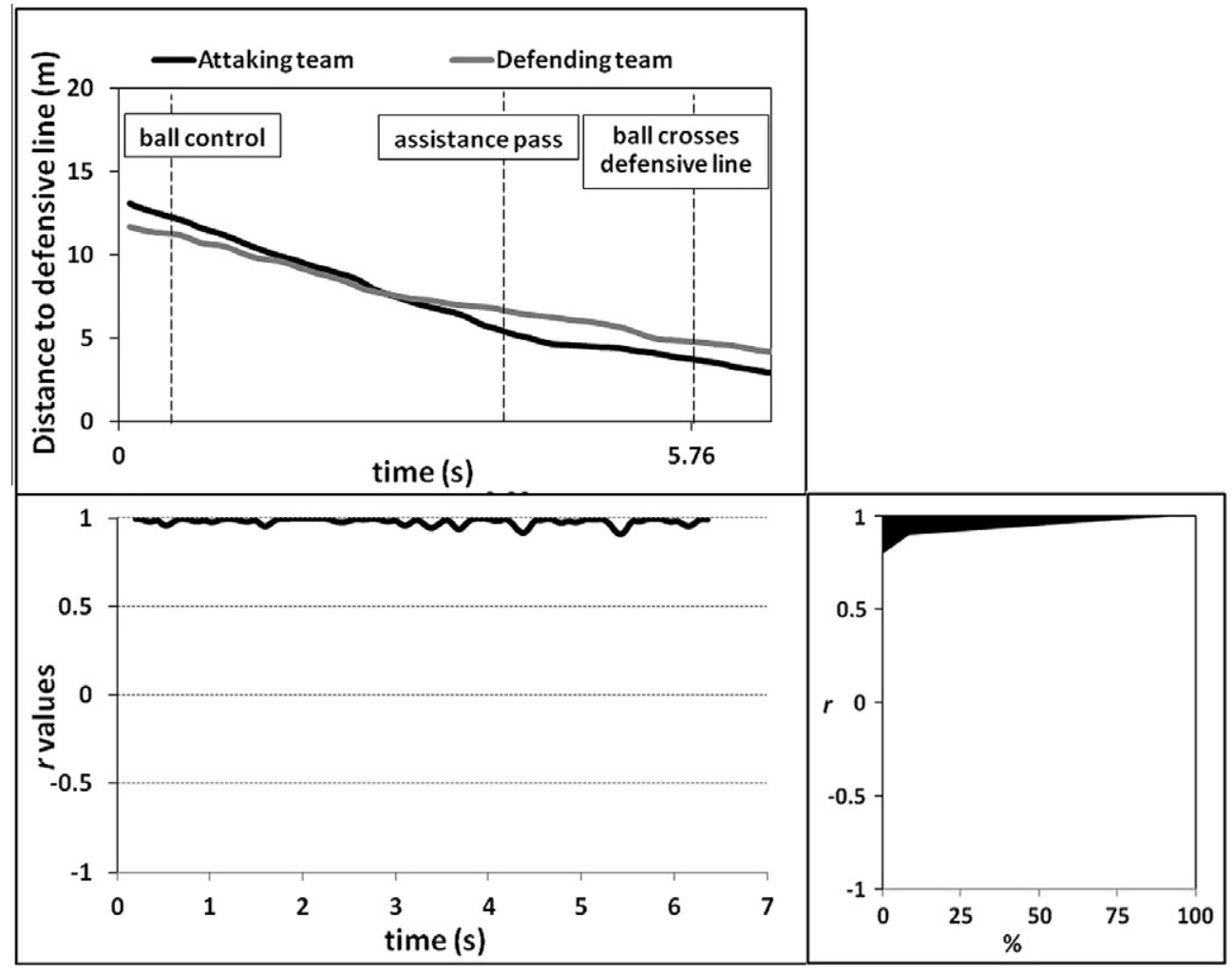

Fig. 2. Exemplar data of coordination tendencies between both teams for centroids during $7 \mathrm{~s}$ of motion in an exemplar play. Top: Variation in the distance of each centroid to defensive line. Bottom: Respective running correlation function and frequency histogram. The tendency of the correlation function to be predominantly positive is captured by the asymmetrical distribution of frequency histogram (bottom right panel). 
Fig. 3 depicts the correlation landscape for the centroids of both teams in all trials. This view of the data highlights the global coordination tendencies between the centroids of the two teams.

The centroid showed predominantly higher correlation values (i.e., values of $r$ near 1 ) for all trials, confirming that the average position of both teams moved consistently at the same time, in the same direction in all the plays.

Fig. 4 presents the variations in surface area of each team in the same random selected play presented in Fig. 2, also evidencing the three key events (upper panel), the corresponding correlation function (bottom left panel) and, frequency histogram (bottom right panel). The data showed that the surface area of each sub-group displayed some fluctuations during the play. Continuous correlation functions also showed high variations between -1 and $1 r$ values, indicating the absence of a clear mode of coordination between the covered areas of the two sub-groups in this play (also observable in the frequency histogram - bottom right panel).

Fig. 5 shows the correlation landscape for the surface areas of both sub-groups in all the plays, evidencing their global coordination tendencies between the two teams.

The continuous correlation function of the surface areas showed fluctuations between the three possible coordinative states (i.e., symmetric, anti-symmetric and uncorrelated states) with no clear predominant coordination tendencies.

\subsection{Sub-group relations at key moments of the plays}

A mixed-model ANOVA revealed that there were differences in the centroid values of both sub-groups of performers at the three key moments of the plays (see Fig. 6), $F(1,38)=21.841$, $p<.001$. Tukey's HSD comparisons revealed that for the attacking team, significant differences were noted between moments of ball control $(M=9.46, S D=3.8)$ and the assisted pass $(M=6.98$, $S D=2.9$ ), and between the moment of ball control and the moment the ball crossed the defensive line $(M=5.27, S D=3.2), p=.001$. For the defending team, Tukey's HSD tests discriminated significant differences between the same moments: ball control $(M=9.01, S D=4.0)$ and assisted pass $(M=7.08$, $S D=3.4), p=.001$, and ball control and ball crossing the defensive line $(M=5.72, S D=3.4), p=.003$. No differences were observed between the centroids of the attacking and defending sub-groups at any time, $F(1,38)=0.002, p>.05)$. However, as noted in Fig. 6 , mean results showed a trend for a crossing of the centroids between the moments of ball control and the assisted pass (i.e., the centroid of the attacking team showed a greater decrease of the distance to the defensive line than the centroid of the defending team). This tendency was confirmed by visual inspection in 13 of 20 trials. In the other seven trials we found a tendency for a decrease in the distance between the centroids of both sub-groups.

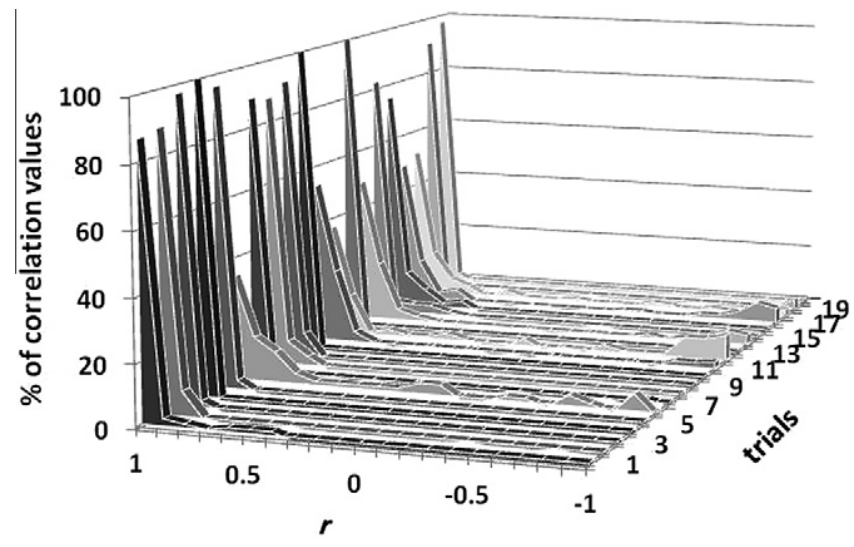

Fig. 3. Correlation landscape for the distance of each centroid to defensive line in all trials $(n=20)$. 


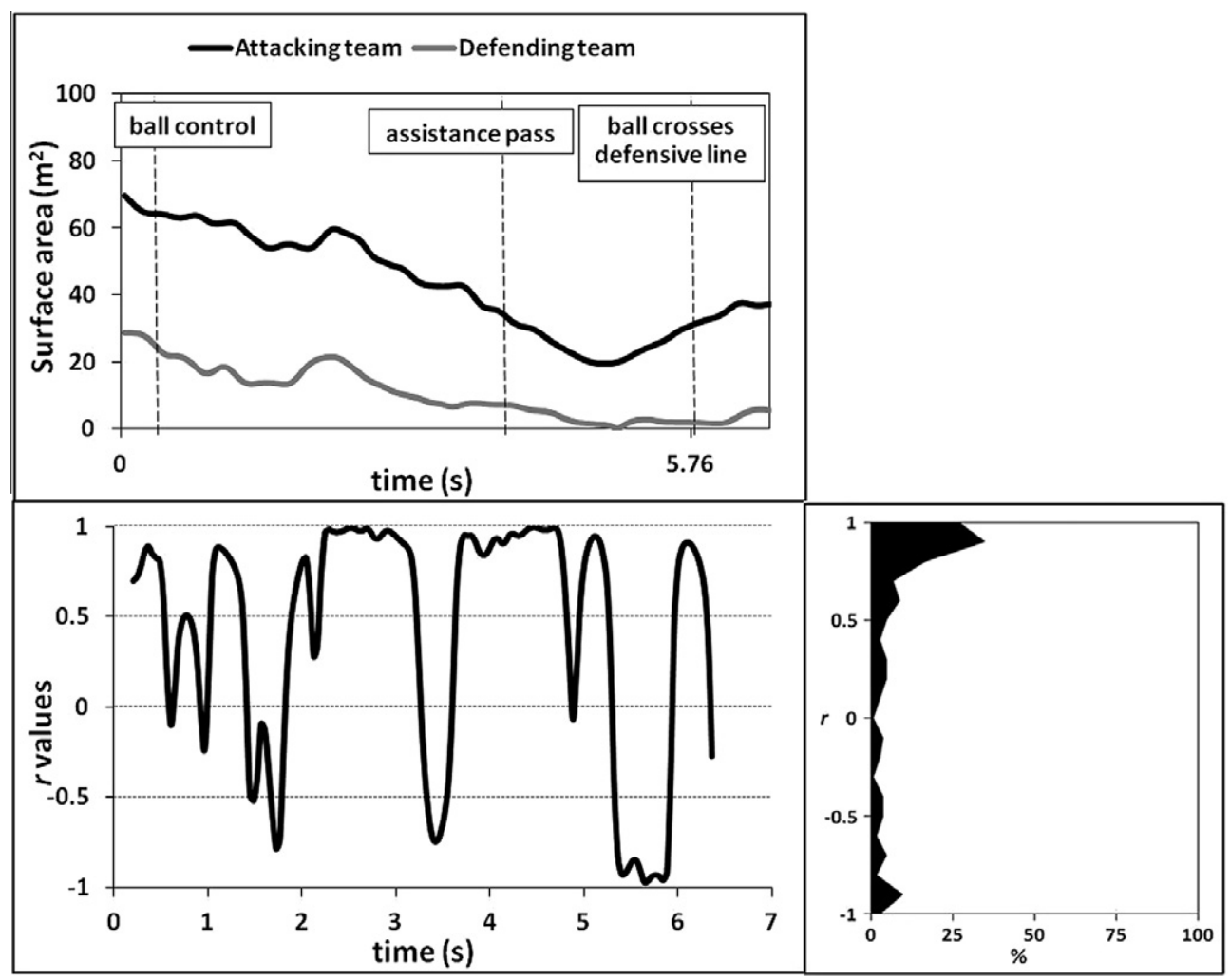

Fig. 4. Exemplar data of coordination tendencies between both teams for surface area during $7 \mathrm{~s}$ of motion. Top: Variation in surface area for both teams. Bottom: Corresponding correlation function and frequency histogram. The fluctuations of the correlation function between positive and negative correlation values resulted in a more equally distributed frequency histogram (bottom right panel).

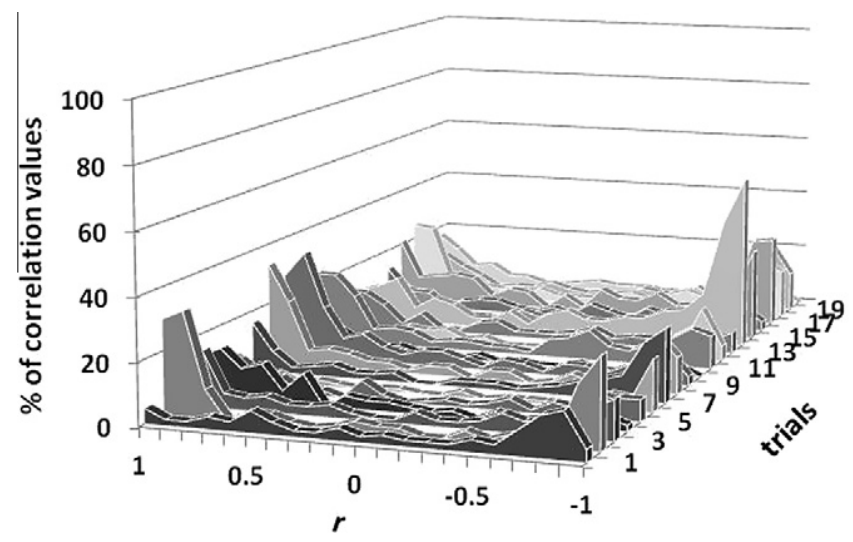

Fig. 5. Correlation landscape for surface area of each team in all trials $(n=20)$.

A mixed-model ANOVA revealed that there were differences in the surface area between attacking and defending sub-groups across the three defined moments (see Fig. 7 ), $F(1,38)=10.086, p \leqslant .003$. 


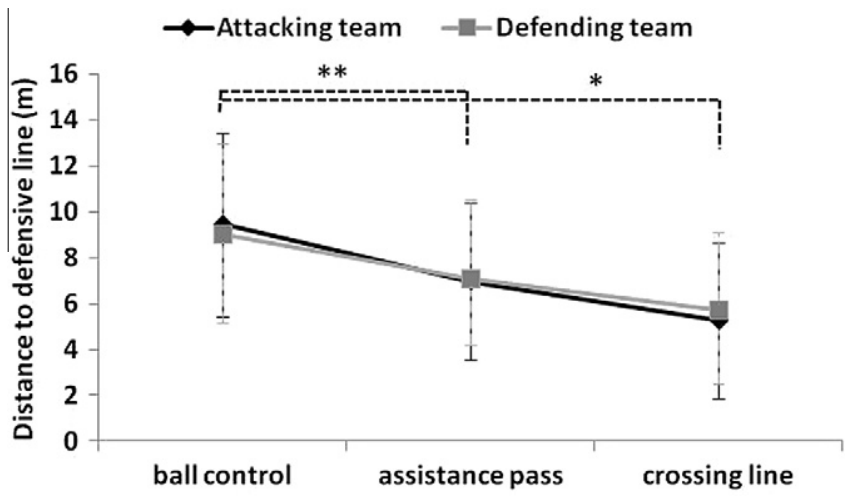

Fig. 6. Distance of the centroid to defensive line for both teams in the three key moments defined. ${ }^{* *}-$ showed statistical differences between ball control and assistance pass moments $(p<.001)$ for both teams; * - showed statistical differences between assistance pass and crossing line moments $(p<.01)$ for both teams. Error bars show standard deviation.

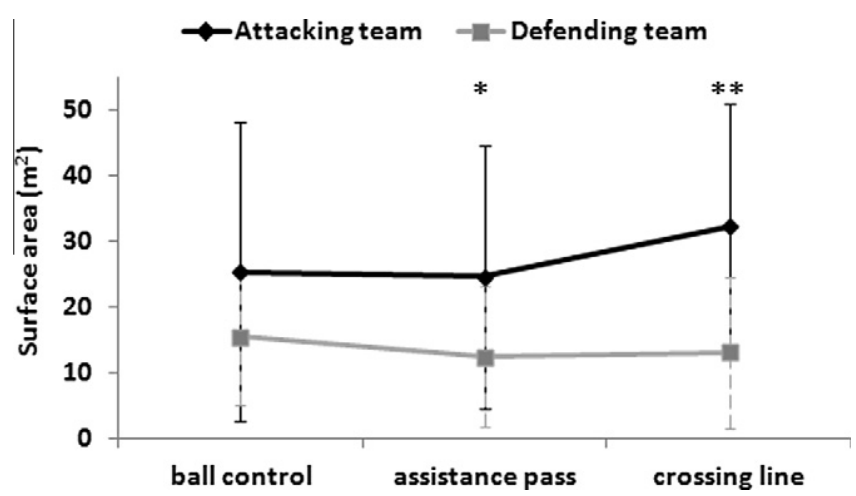

Fig. 7. Surface area for both teams in the three key moments defined. ${ }^{*}$ - showed statistical differences between attacking and defending teams in the passing moment $(p=.018)$; ${ }^{* *}$ - showed statistical differences between attacking and defending teams in the moment that the ball crosses defensive line $(p=.001)$. Error bars show standard deviation.

Tukey's HSD comparisons revealed that the differences were only for the moment of the assisted pass (attacking team: $M=24.75, S D=20.0$, defending team: $M=12.55, S D=10.6, p=.018$ ) and for the moment when the ball crossed the defensive line (attacking team: $M=32.32 ; S D=18.8$; defending team: $M=13.18 ; S D=11.6 ; p=.001$ ). No differences in surface area were observed within the three key moments of performance $(F(1,38)=1.345, p>.05)$.

\section{Discussion}

The main goal of this study was to identify how coordination tendencies between team players emerged from collective system behaviors in 3 vs 3 sub-phases of performance near the scoring zone in the team sport of football. To achieve this aim, the group-motion variables 'centroid' and 'surface area' were analyzed to capture the interactions within and between two sub-groups of players. We found that the emerging coordination tendencies exhibited a predominantly symmetric pattern between the centroid of the teams in all trials. Despite the fluctuations in centroid displacement time-series, results showed that the average position of both teams approached and moved away from a defensive line in a highly coupled fashion as demonstrated by high positive correlation values. This 
compound physical variable elegantly captured the rhythmic flow of attacking and defending patterns of play in 3 vs 3 sub-phases near the scoring zone, in which all the players seemed to move collectively. These results are in agreement with the findings of Frencken and Lemmink (2008) and Frencken et al. (2011) in the team sport of football, and Bourbousson, Seve, and McGarry (2010) in basketball, who observed a high synchronization between team centers in the longitudinal plane of the pitch. However, in the present study, we used a representative practice task that allowed us to capture the coordination dynamics of opposing sub-groups in a specific performance setting where performers tried to create/prevent shooting opportunities (Araújo, Davids, \& Passos, 2007; Davids, Button, Araújo, Renshaw, \& Hristovski, 2006; Dicks, Davids, \& Araújo, 2008).

Results from the statistical analyses showed significantly superior centroid mean values at the moment of ball control by the passing player, compared with the moment of the assisted pass and the moment of the ball crossing the defensive line in both teams. Statistical analyses also showed no significant differences between the distance from the attacking and defending sub-groups' centroids to the defensive line. This finding implies that both teams progressively approached the scoring zone, with high proximity between the centers of the teams at almost all times during competitive performance. Curiously, as Fig. 6 shows, there is a mean tendency for a crossing, or at least a decrease in the distance, of the centroids of the teams in the immediate instants before the time of the assisted pass. This mean tendency for centroids to cross or, at least, to approach each other, was confirmed by individual inspection for all trials. In an 11-a-side game format, Lames et al. (2010) found an almost perfect synchrony between the centroids of the two teams during the entire match studied. Relating the findings of that study to our data, it may be suggested that the approaching of the centroids reported in the present study might be evidence that transitions at this level of system organization (e.g., as a 3 vs 3 sub-phase nears a scoring zone) are fundamental for a loss of system stability during collective competitive performance (i.e., 11 vs 11 ). This may be one of the main perturbations (McGarry et al., 2002) that might change the organizational state of a competitive match. However, literature suggests that there was not a linear relationship between these transitions at a micro- and macro-level analysis of the system (Gréhaigne et al., 1997). These non-linear relationships between different levels of analysis demonstrate the functional role of (micro)variability in (macro)system dynamics (Davids et al., 2005, 2008). This feature of teams' centroid measures to approach each other before the final pass in the move was a consequence of the high rate of change in the distance of the attacking team to the defensive line. This outcome was achieved as a result of stable tendencies in coordination between players within and between each team. Despite the high variability in the individual behaviors of attacking players (e.g., creating support, running along the pitch or creating space for teammates), and defending players (e.g., marking an opponent, covering a teammate, controlling space), the collective patterns of behavior were very stable among all the trials. As reported by Frencken and Lemmink (2008) and Frencken et al. (2011), these data might suggest that the loss of stability in the 3 vs 3 subphases seems to be related to a previous crossing in the centroids of the teams, or at least, to an approaching between them. In the present study we found that the emergence of the moment of the assisted pass was related to specific spatiotemporal relations between the two sub-groups of players.

For the surface area measure, there were no clear tendencies in running correlation values observed in all trials. The very unstable mode of coordination was characterized by the highly variable fluctuations in correlation functions over time. This feature of performance was indicative of no prevalent pattern of coordination between teams for this compound group-motion variable. In 3 vs 3 football sub-phases of play, it seems that teams increase or decrease their surface area independently of the behaviors of the opposing team. These data showed that the surface area had limited capacity to capture the coordination dynamics between these two sub-groups of players near the scoring zone, and confirmed the proposals of Frencken and Lemmink (2008) and Frencken et al. (2011) with regards to this variable. However, it may be hypothesized that variations in surface area of each team are the result of coordination tendencies emerging within each team, constrained by the functional relations between their own players during the approach to the scoring zone. Results from a mixed-model ANOVA confirmed these expectations, by showing that differences between teams progressively increased along the three key moments of performance (i.e., ball control, assisted pass and crossing line), despite the absence of prevalent patterns of coordination between teams in the correlation landscape. This finding can be indicative of within-group coordination processes, in which attacking 
players coordinated their actions in order to increase team space to move into the scoring zone, with some degree of independence from the opposing group of performers. Focusing on the post hoc tests results, we found that the differences were observed only at the moments of the assisted pass and when the ball crossed the defensive line. These findings might suggest the importance of increasing the surface area to the attacking sub-groups in order to destabilize the opposing team and to create shooting opportunities (Gréhaigne et al., 1997), but only immediately before the moment of the assisted pass. However, in this study we did not compare successful and unsuccessful plays to better support this explanation. This is an issue for further research. The apparent lack of surface area to describe the coordination between the sub-groups near the scoring zone may not be transferable to other sub-phases of play or the context of the full match. For example, it might be argued that sudden changes in surface area of the teams at match level might be indicative of exchanges in ball possession. In this study, we investigated only plays without exchanges in ball possession, and these propositions need to be tested empirically.

To summarize, in this study, we investigated the collective patterns of behavior in 3 vs 3 subphases of play in a representative context of creation/prevention of goal scoring opportunities. The centroids of the sub-groups demonstrated a strong symmetric relation that described the collective attacking/defending performers' behaviors in this sub-phase near the scoring zone. This relation showed a mean tendency for an approaching (even a crossing) of the centroids immediately before the loss of stability in the system (i.e., the assisted pass). The surface area did not show a clear coordination pattern between teams. However, it revealed that the difference in the occupied area between the attacking and defending teams also significantly increased immediately before the assisted pass was made. The between- and within-team coordination tendencies reported for these compound group-motion variables allowed an understanding of the dynamics of the collective behaviors in this typical competitive performance situation. Results also emphasized that major changes in sub-group behaviors occurred just before an assisted pass was made in the performance sub-phase.

The current study showed how interpersonal coordination processes within and between two small groups of competing football players can be captured by compound physical variables that synthesized the functional relationships between individuals and the performance environment. The time-evolved group behaviors described in this study were related to discrete game events considered

1. Schematic representation of single camera video motion capture.

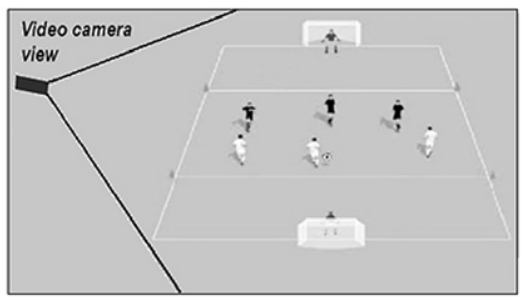

4. Converted pitch coordinates ( $m$ ) allowed to reproduce movement displacement trajectories of players in the space of action.
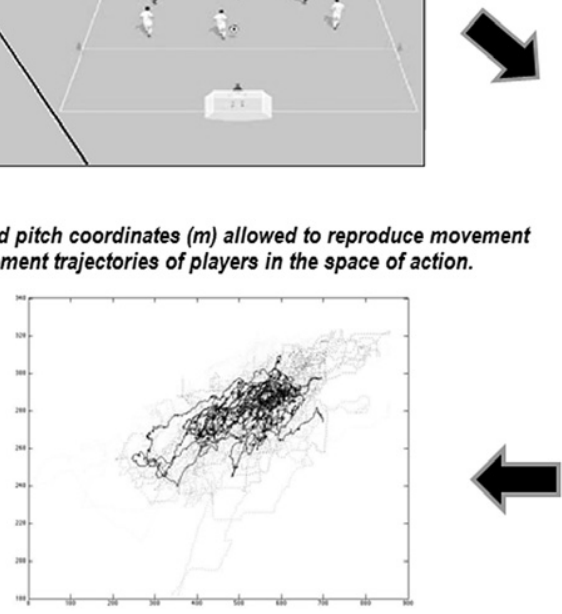

Fig. 8. Flow chart representation of the time-motion analysis procedures employed.
2. TACTO 8.0 device window: manual tracking of a selected working point with computer mouse allow to obtain virtual coordinates of the tracked player/object.

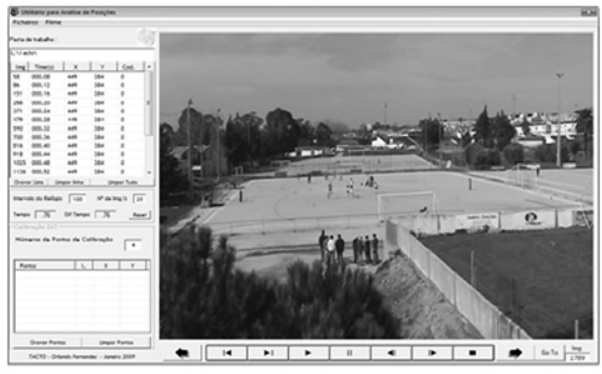

3. Direct linear transformation (2D-DLT) method for camera calibration and bi-dimensional reconstruction.
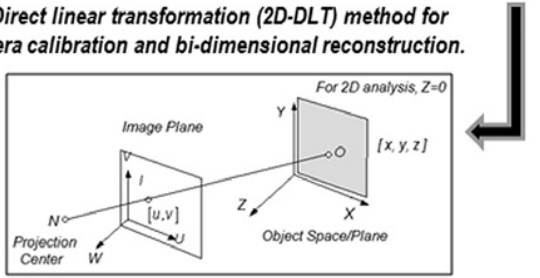
as influential in breaking the initial stability of the relative positioning of the two sub-groups. The current investigation used methods and tools that can be applied to develop a deep understanding of interpersonal coordination processes in other team sports and in other social collectives where continuous interactions between people is an important issue and has a crucial meaning.

\section{Acknowledgments}

The first author was supported by a doctoral grant of the Portuguese Foundation for Science and Technology (SFRH/BD/43994/2008).

\section{Appendix A}

Time-motion analysis procedures used in the current study involved manual video tracking and bidimensional reconstruction, using a single video camera. Here, we briefly describe the sequential steps of the method.

1. Data collection - The first step consisted of recording the participants' behaviors using a regular digital video camera positioned statically at $30 \mathrm{~m}$ from the pitch. It was placed at $5 \mathrm{~m}$ height, perpendicular to the longitudinal component of the pitch and with an elevation angle of approximately $10^{\circ}$. Before the start of the experiment, several non-collinear control points (corresponding to specific landmarks visible in the video camera) were measured for later calibrations.

2. Image treatment - The software package TACTO 8.0 (Fernandes et al., 2010) was used to extract the positional coordinates (pixels units) from participants' movement displacement trajectories. The procedure consisted of following with a computer mouse cursor a working point located between the feet of each participant. This working point was used because it represents the projection of the player's center of gravity on the ground (Duarte et al., 2010). The TACTO package was also used to assess the virtual coordinates of the seven control points selected that afterwards were used for calibration.

3. Camera calibration and bi-dimensional reconstruction - Camera calibration and object-plane reconstruction were done using the bi-dimensional Direct Linear Transformation (2D-DLT) method (Abdel-Aziz \& Karara, 1971; Kwon, 2008). This two-dimensional method uses the same DLT algorithms employed in tri-dimensional analysis, but considers the $z$-coordinates always equal to zero. The DLT method is considered also to deal with some measurement errors reported by other methods such as optical distortion and de-centering distortion (Marzan \& Karara, 1975). The DLT method directly relates an object point located in the object space/plane and the corresponding image point on the image plane from the camera. Two reference frames are defined object-space reference frame (the $X Y Z$-system) and image-plane reference frame (the $U V$-system) (see bottom left panel of Fig. 8). The $[x, y, z]$ is the object-space coordinates of point $O$, while $[u, v]$ is the image-plane coordinates of the image point $I$. There is a direct relationship between the object space coordinates, $[x, y, z]$, and the image plane coordinates, $[u, v]$, as shown in Eqs. (A1) and (A2):

$$
\begin{aligned}
& u_{i}-u_{o}-\Delta u_{i}=-\lambda_{u} w_{o} \cdot \frac{t_{21}\left(x_{i}-x_{o}\right)+t_{22}\left(y_{i}-y_{o}\right)+t_{23}\left(z_{i}-z_{o}\right)}{t_{11}\left(x_{i}-x_{o}\right)+t_{12}\left(y_{i}-y_{o}\right)+t_{13}\left(z_{i}-z_{o}\right)} \\
& v_{i}-v_{o}-\Delta v_{i}=-\lambda_{v} w_{o} \cdot \frac{t_{31}\left(x_{i}-x_{o}\right)+t_{32}\left(y_{i}-y_{o}\right)+t_{33}\left(z_{i}-z_{o}\right)}{t_{11}\left(x_{i}-x_{o}\right)+t_{12}\left(y_{i}-y_{o}\right)+t_{13}\left(z_{i}-z_{o}\right)}
\end{aligned}
$$

where $i$ is the control point number, $\left[0, u_{i}, v_{i}\right]$ and $\left[w_{o}, u_{o}, v_{o}\right]$ are the image plane coordinates of the image point $(I)$ and the projection center $(N)$, respectively, $\left[x_{i}, y_{i}, z_{i}\right]$ and $\left[x_{0}, y_{0}, z_{0}\right]$ are the object space/plane coordinates of the object point $(O)$ and the projection center $(N)$, respectively, $\left[\Delta u_{i}, \Delta v_{i}\right]$ are the optical errors (optical distortion and de-centring distortion, Marzan \& Karara, 1975) involved in the image coordinates, and $\left[\lambda_{\mathrm{u}}, \lambda_{\mathrm{v}}\right]$ are the scaling factors for the unit conversion from the real-life unit to the digitizer unit (DU). The $t_{11}-t_{33}$ in Eqs. (A1) and (A2) are the elements of a $3 \times 3$ transformation matrix from the object-space/plane reference frame to the image-plane reference frame. 
Successive rearrangements of Eqs. (A1) and (A2) resulted in 11 DLT parameters that reflect the relationships between the object-space/plane reference frame and the image-plane reference frame. In the current study, due to the utilization of planar analysis, DLT parameters were reduced to 8. Using the virtual and pitch coordinates of the 7 control points, we calculated the DLT parameters used for camera calibration and image reconstruction procedures according the algorithms presented in Woltring and Huiskes (1990).

\section{References}

Abdel-Aziz, Y. I., \& Karara, H. M. (1971). Direct linear transformation from comparator coordinates into object space coordinates in close-range photogrammetry. In Proceedings of the symposium on close-range photogrammetry (pp. 1-18). Falls Church, VA: American Society of Photogrammetry.

Araújo, D., Davids, K., \& Hristovski, R. (2006). The ecological dynamics of decision making in sport. Psychology of Sport and Exercise, 7, 653-676.

Araújo, D., Davids, K., \& Passos, P. (2007). Ecological validity, representative design, and correspondence between experimental task constraints and behavioral setting: Comment on Rogers, Kadar, and Costall (2005). Ecological Psychology, 19, 69-78.

Bar-Yam, Y. (2004). Making things work: Solving complex problems in a complex world. Cambridge, MA: New England Complex Systems Institute, Knowledge Press.

Beek, P. J., Peper, C. E., \& Stegeman, D. F. (1995). Dynamical models of movement coordination. Human Movement Science, 14, 573-608.

Beek, P. J., Verschoor, F., \& Kelso, J. A. S. (1997). Requirements for the emergence of a dynamical social psychology. Psychological Inquiry, 8, 100-104.

Bourbousson, J., Seve, C., \& McGarry, T. (2010). Space-time coordination dynamics in basketball: Part 2. The interaction between the two teams. Journal of Sports Sciences, 28, 349-358.

Corbetta, D., \& Thelen, E. (1996). The developmental origins of bimanual coordination: A dynamic perspective. Journal of Experimental Psychology: Human perception and Performance, 22, 502-522.

Davids, K., Araújo, D., \& Shuttleworth, R. (2005). Applications of dynamical systems theory to football. In T. Reilly, J. Cabri, \& D. Araújo (Eds.), Science and football $V$ (pp. 547-550). Oxon: Routledge.

Davids, K., Button, C., Araújo, D., Renshaw, I., \& Hristovski, R. (2006). Movement models from sports provide representative task constraints for studying adaptive behavior in human motor systems. Adaptive Behavior, 14, 73-95.

Davids, K., Button, C., \& Bennett, S. (2008). Dynamics of skill acquisition. A constraints-led approach. Champaign: Human Kinetics.

Deneubourg, J.-L., \& Goss, S. (1989). Collective patterns and decision-making. Ecology, Ethology and Evolution, 1, $295-311$.

Dicks, M., Davids, K., \& Araújo, D. (2008). Ecological psychology and task representativeness: Implications for the design of perceptual-motor training programmes in sport. In Y. Hong \& R. Bartlett (Eds.), Routledge handbook of biomechanics and human movement science (pp. 129-139). New York, NY: Routledge.

Duarte, R., Araújo, D., Fernandes, O., Fonseca, C., Correia, V., Travassos, B., et al (2010). Capturing complex human behaviors in representative sports contexts with a single camera. Medicina-Lithuania, 46, 408-414.

Farkas, I. J., \& Vicsek, T. (2005). Patterns in the collective behavior of humans. AIP Conference Proceedings, 779, 1-15.

Fernandes, O., Folgado, H., Duarte, R., \& Malta, P. (2010). Validation of the tool for applied and contextual time-series observation. International Journal of Sport Psychology, 41, 63-64.

Frencken, W., \& Lemmink, K. (2008). Team kinematics of small-sided soccer games. A systematic approach. In T. Reilly \& F. Korkusuz (Eds.), Science and football VI (pp. 161-166). New York, NY: Routledge.

Frencken, W., Lemmink, K., Delleman, N., \& Visscher, C. (2011). Oscillations of centroid position and surface area of soccer teams in small-sided games. European Journal of Sport Science, 11, 215-223.

Gréhaigne, J. F., Bouthier, D., \& David, B. (1997). Dynamic-system analysis of opponent relationships in collective actions in soccer. Journal of Sports Sciences, 15, 137-149.

Hughes, M., \& Franks, I. M. (2005). Analysis of passing sequences, shots and goals in soccer. Journal of Sports Sciences, 23, 509-514.

Kelso, J. A. S. (1984). Phase transitions and critical behavior in human bimanual coordination. American Journal of Physiology: Regulatory, Integrative and Comparative, 15, 1000-1004.

Kugler, N. P., Kelso, J. A. S., \& Turvey, M. T. (1980). On the concept of coordinative structures as dissipative structures: I. Theoretical lines of convergence. In G. E. Stelmach \& J. Requin (Eds.), Tutorials in motor behavior (pp. 3-47). New York: North-Holland Publishing.

Kwon, Y. H. (2008). Measurement for deriving kinematic parameters: Numerical methods. In Y. Hong \& R. Bartlett (Eds.), Handbook of biomechanics and human movement science (pp. 156-181). Abingdon, Oxon.

Lames, M., Erdmann, J., \& Walter, F. (2010). Oscillations in football - Order and disorder in spatial interactions between the two teams. International Journal of Sport Psychology, 41, 85-86.

Mallon, E. B., Pratt, S. C., \& Franks, N. R. (2001). Individual and collective decision-making during nest site selection by the ant Leptothorax albipennis. Behavioural Ecology and Sociobiology, 50, 353-359.

Marzan, G. T., \& Karara, H. M. (1975). A computer program for direct linear transformation solution of the collinearity condition, and some applications of it. In E. E. Daniel (Ed.), Proceedings of the symposium on close-range photogrammetric systems (pp. 420-476). Falls Church, VA: American Society of Photogrammetry.

McAvoy, N. (1998). Teaching soccer fundamental - A progressive guide to maximize player development. Champaign, IL: Human Kinetics.

McGarry, T., Anderson, D., Wallace, S., Hughes, M., \& Franks, I. (2002). Sport competition as a dynamical self-organizing system. Journal of Sports Sciences, 15, 171-181.

McGarry, T. (2005). Soccer as a dynamical system: Some theoretical considerations. In T. Reilly, J. Cabri, \& D. Araújo (Eds.), Science and football $V$ (pp. 551-560). Oxon: Routledge. 
Meador, K. J., Ray, P. G., Echauz, J. R., Loring, D. W., \& Vachtsevanos, G. J. (2002). Gamma coherence and conscious perception. Neurology, 59, 847-854.

Moorhouse, K. M., \& Granata, K. P. (2007). Role of reflex dynamics in spinal stability: Intrinsic muscle stiffness alone is insufficient for stability. Journal of Biomechanics, 40, 1058-1065.

Palut, Y., \& Zanone, P.-G. (2005). A dynamical analysis of tennis: Concepts and data. Journal of Sports Sciences, 23, 1021-1032.

Partridge, B. L. (1982). The structure and function of fish schools. Scientific American, 246, 90-99.

Partridge, B. L., \& Pitcher, T. J. (1979). Evidence against a hydrodynamic function for fish schools. Nature, 279, 418-419.

Passos, P., Araújo, D., Davids, K., Gouveia, L., Serpa, S., Milho, J., et al (2009). Interpersonal pattern dynamics and adaptive behavior in multiagent neurobiological systems: Conceptual model and data. Journal of Motor Behavior, 41, 445-459.

Pratt, S. C., Mallon, E. B., Sumpter, D. B., \& Franks, N. R. (2002). Quorum-dependent recruitment and collective decision-making during colony emigration by the ant Leptothorax albipennis. Behavioural Ecology and Sociobiology, 52, 117-127.

Rampinini, E., Impellizzeri, F. M., Castagna, C., Abt, G., Chamari, K., Sassi, A., et al (2007). Factors influencing physiological responses to small-sided soccer games. Journal of Sports Sciences, 25, 659-666.

Schöllhorn, W. (2003). Coordination dynamics and its consequences on sports. International Journal of Computer Science in Sport, $2,40-46$.

Visscher, P. K., \& Camazine, S. (1999). Collective decisions and cognition in bees. Nature, 397, 400.

Winter, D. A. (2005). Biomechanics and motor control of human movement (3rd ed.). New York: John Wiley \& Sons.

Woltring, H. J., \& Huiskes, R. (1990). Stereophotogrammetry. In N. Berme \& A. Capozzo (Eds.), Biomechanics of human movement (pp. 108-127). Worthington, OH: Bertec Corporation.

Yuan, J., Wang, J., Xu, Z., \& Li, B. (2005). Time-dependent collective behavior in a computer network model. Physica A: Statistical Mechanics and its Applications, 368, 294-304. 